\title{
Research of Imaging Simulation System Based on Target near Field Echoes
}

\author{
DENG Wei ${ }^{\mathrm{a}}$, ZHENG Chenyao $^{\mathrm{b}}$, WU Helong ${ }^{\mathrm{c}}$ \\ 93 unit of 91388 Army, Zhan Jiang of Guang Dong 524022 \\ achenlizhidengwei@163.com, bhchy@foxmail.com, ${ }^{\mathrm{b}}$ qingwu321@yeah.net
}

Keywords: the planar element method; multi-beam image; acoustic highlights

\begin{abstract}
The theory and function of mathematical imaging simulation system based on underwater multi-targets were introduced in this paper. The accuracy and feasibility of the simulation system that had a good image display function and the dynamic simulation function with OpenGL graphics library were provided through specific examples and experiments. It would generate important value in engineering to provide theoretical analysis basis for echoes characteristics of the underwater targets.
\end{abstract}

\section{Introduction}

Acoustic was applied in the military at the earliest stage, and played a key role in two wars. The effect of acoustic in water can not be replaced by other physical fields that human has known at present. Echoes of underwater target contains abundant information of characteristic to underwater target, such as target material, the target size and structure...etc. It was important criterion for sonar and torpedo to detect targets, and to distinguish true or false targets. Therefore, it was importance to forecasting characteristics of target in underwater. In recent years, Acoustic imaging was extensively by human used to carry on to probe into, investigate to explore, position in water, the target identify, navigate, correspond by letter, telemetering control etc. Along with micro-electronics technique quickly development and calculator operate the exaltation of velocity, voice the wave became to be like a technique to get an extensive application while being solid, voice wave several bunches are also $3 \mathrm{D}$ to become to be like a technique to not only get the directions, distance of underwater object, but can also get the clear outline of object, this carries on underwater object to probe into, identify to bring a tremendous progress for the noise wave.

\section{Method and principles}

The imaging Simulation System Based on target in near field echo according to the physical acoustics and the planar element method $^{[1 \sim 3]}$ was developed. Physical acoustic (Kirchhoff approximation) method is the most commonly method in the engineering. Kirchhoff regarded as the size of the size of target was the more long than wave-length, and the curvature radius of this noodles was also larger than wave-length, then could think that the ratio of superficial wave velocity and vibrate velocity from target surface looks like planar wave. Thus the Helmholtz-Kirchhoff integral calculus equation was simplified into an area to divide that was tremendous simplification. It is thus clear that Kirchhoff approximation is similar a kind of high frequency planar wave. Surface integral was leaded by Kirchhoff approximation for the brief convex smooth surface can make use of steady mutually integral calculus method(the saddle orders a method) to get spread shoot a field of resolution solution, and for complex targets could make use of number integral calculus method to get scattered field solution. Kirchhoff approximation was leaded by the rigid surface at the beginning, but it was expanded to non-rigid surface ${ }^{[4]}$ later. 


\section{System functions introduction}

(1)Imaging simulation of two-dimensional space echoes and fine mathematics model of target were established by the system, and the Submarine and torpedo model were pre-stored in the system;

(2)The point target, scale target and noise model of target were established by the system;

(3)Model of target in Ocean acoustic channel (consideration sea surface and bottom of sea) was established by the system, and the depth of sea and parameters were defined by the customers;

(4)Echoes of a period signal sequence can be imitated, and parameter of shooting signal defined by the customers;

(5)Acoustic imaging of single target and multiple targets could be imitated by the system;

(6)Dynamic imaging could be simulated by the system;

(7)Echoes acoustic highlights strength ${ }^{[5]}$ and the space directions data document could be generated. The interface of the system simulation was shown in figure 1.

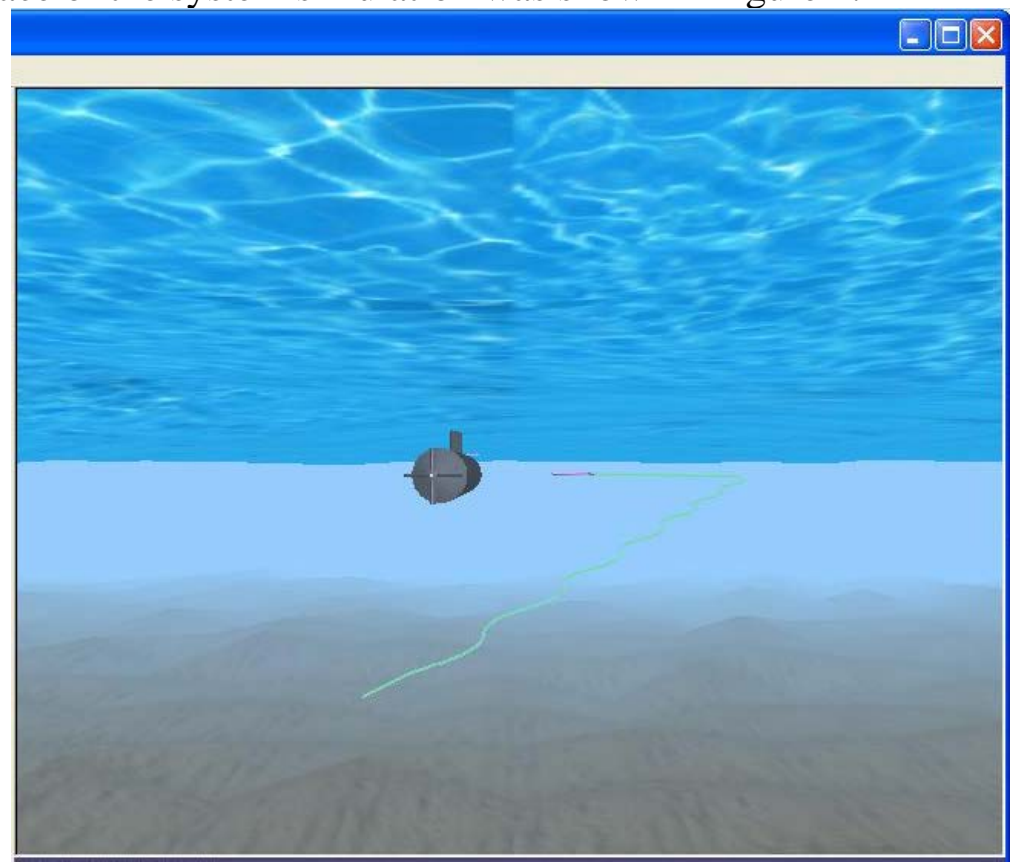

Fig.1 Interface of the system simulation

\section{Simulation flow}

The space wave beam was generated by sampling space scope signal and using mutually moving parameter to adjust a sample data. The t time-domain beam formation was that radicle in each change ability machine of the exportation compensated while tying a direction to postpone by the wave together and mutually, then mutually added the wave of getting different direction bunch energy. The expression of time-domain beam is:

$$
b(t, \boldsymbol{u})=\left|\sum_{m=0}^{M-1} \sum_{n=0}^{N-1} \omega_{m, n} S_{m, n}(t-\tau(\boldsymbol{u}, m, n))\right| .
$$




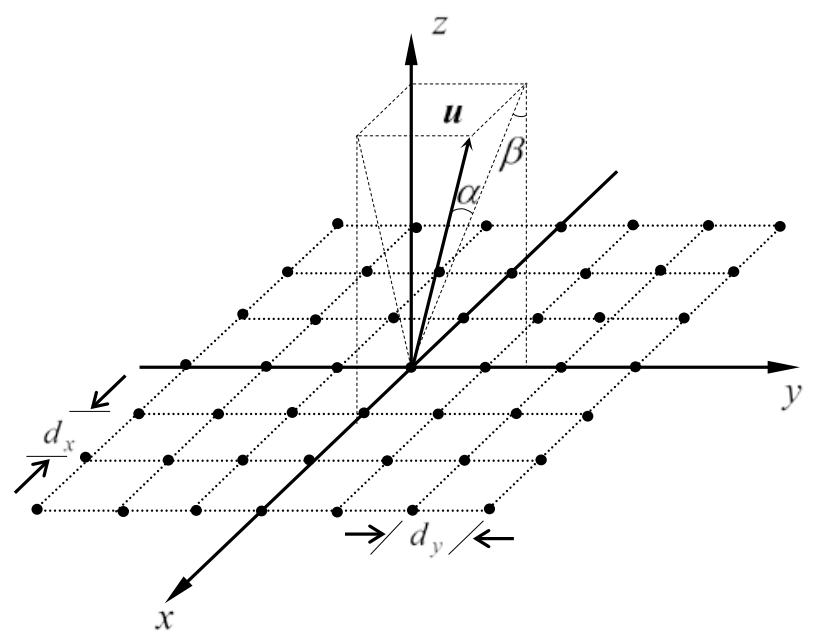

Fig.2 Bunch formation the space set up

The wave bunch formation of the realization could have various methods, such as time delay to sum up bunch formation, Frequency beam-forming etc. Especially, the direction of the signal arrive will unknown for acoustic imaging in underwater and acoustic homing torpedo in real. Consequently, searching quickly targets must form a set of beams to cover possible cape dimension. The time delay is only integer multiples to sampling interval for number system. The cost of the whole hardware of system might up if sample frequency is very high. In order to simplify the system and and improve system speed, the system used quadrature beam-forming way to reduce the sampling frequency. The diagram based on quadrature beam-forming is shown in figure 3.

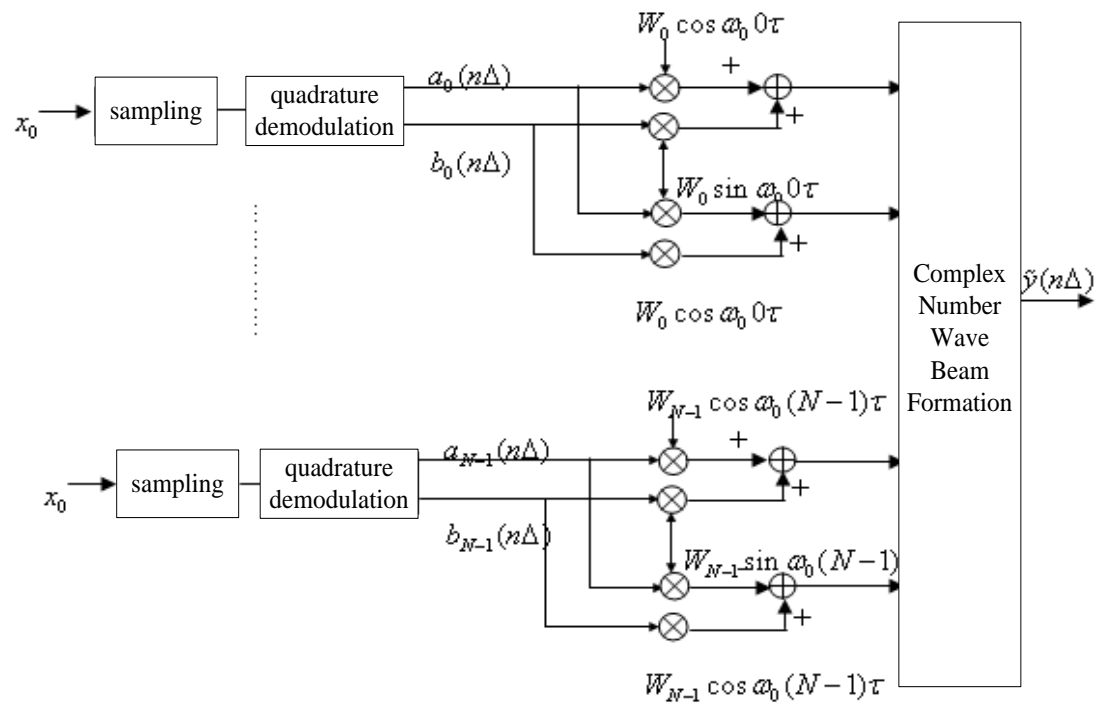

Fig.3 The diagrams based on quadrature beam-forming

\section{Mathematical imaging model of the target 3D acoustic figure}

Straight array can search sector only when torpedo is homing, namely form some searching beam in single horizontal planar. The beam is symmetrical in the vertical direction, thus it cannot distinguish two targets up there. In order to determine pitch angle of targets in the vertical planar, we must collocate necessary beam levels and at least two, then distinguish the direction of the torpedo which is up or down. On the other hand, for the sake of distinguish multi-target in the vertical planar, we must ensure a constant searching sector, that is to say collocate multi-level beam. When the level is more, the function of resolution is better. So the homing torpedo system will have large ability of space searching only by collocating 3D multi-beam in the planar array.

The planar array ${ }^{[6]}$ can carry out deflection and scansion of acoustic beam in both horizontal and vertical directions which extensively applies to ultrasound ${ }^{[7]}$, homing torpedo ${ }^{[8]}$ etc. The typical 
sonar Echo Scope 1600 in the planar array image is combined with $40 \times 40$ planar array which has 1600 array elements. Operating frequency is $150 \mathrm{kHz}, 300 \mathrm{kHz}$ and $600 \mathrm{kHz}$, direction resolution ratio is $64 \times 64$. As is well known, the straight array can distinguish horizontal highlights of targets, but not vertical highlights. For resolving this problem, we discuss the planar array application of target echo 3D imaging.

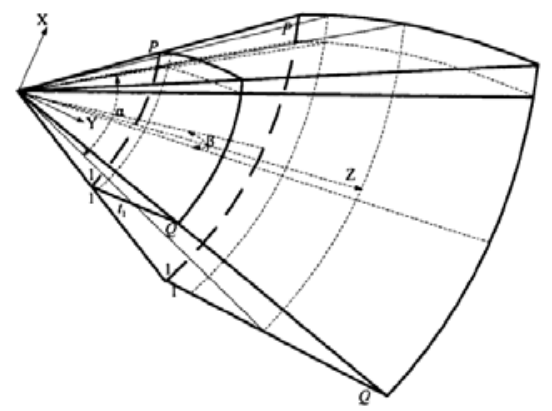

Fig.4 3D beam forming by the planar array

\section{The calculating example verification of simple targets}

In order to validate the validity of multi-beam acoustic imaging, it gives three measuring direction results of sphere targets planar beam forming at different positions. We receive echo of sphere (1), (2) and (3) by a $40 \times 40$ planar array fixing with half wave-length. The echo is calculated by the planar element method, the space position of planar array and three spheres is shown in figure 4 . The coordinate position of acoustic source is $(0,0,0)$, signal frequency is $f_{0}=30 \mathrm{kHz}$, sampling frequency is $f_{\mathrm{s}}=120 \mathrm{kHz}$. Center coordinate of sphere (1) is $\left(50,-23^{0}, 19^{0}\right)$, radius is $r_{2}=3$. Center coordinate of sphere (3) is $\left(60,5^{0},-25^{0}\right)$, radius is $r_{3}=2$.

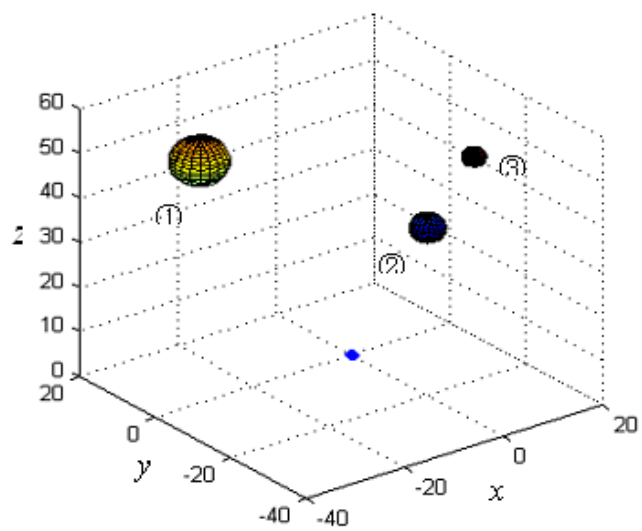

Fig.4 The space position of sphere

The simulation of planar array multi-beam is shown in figure 5 . The imaging result of target sphere is identical with its location which demonstrates the feasibility of the algorithm.

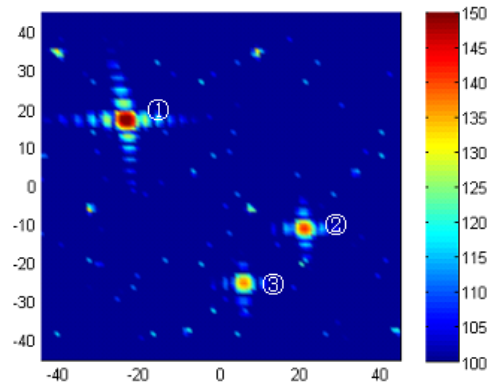

Fig.5 The acoustic imaging of spheres

\section{Imaging simulation of submarine targets}

The imaging simulation system of target short-range echo primarily points at the simulation of underwater complex target. In this paper simulate an example of the complex Benchmark submarine. If we receive the target echo signal by a $40 \times 40$ planar array fixing with half wave-length. The 
acoustic source is located at center of the planar array, center frequency is $f_{0}=30 \mathrm{kHz}$, sampling frequency is $f_{\mathrm{s}}=120 \mathrm{kHz}$. The target towards the planar array at a slant direction of $45^{\circ}$. We calculate beam forming of target echo signal, figure 6 is the relationship of submarine and base-array position.

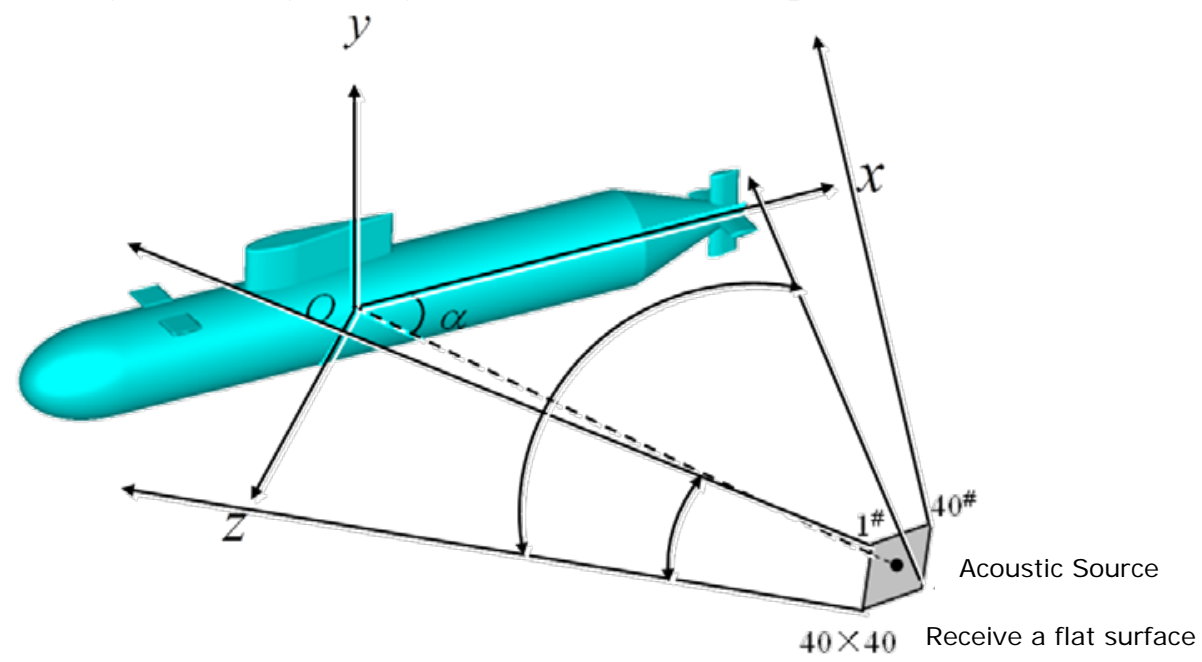

Fig.6 The relationship of submarine and base-array position

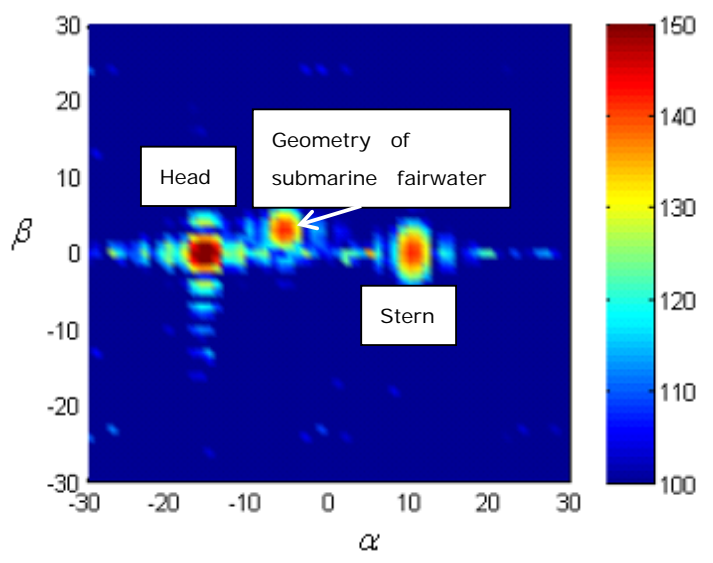

Fig.7 An acoustic image of submarine

Figure 7 is an acoustic image of submarine under planar array with multi-beam. It is shown that Benchmark submarine model target contains three highlights such as head, round-hull, and tail, where the pitch angles of head and tail both are 0 degree, and that round-hull has a constant pitch angle. The highlights of head and submarin sail approaches on the space direction which is corresponding to the structure of target model.

\section{Conclusions}

"The mathematical imaging simulation system based on target near field echoes" adopts echo characteristic model technique of the planar element method to achieve a 3-D display of the submarine model combined with OpenGL figure database. The simulation system carries out functions of an accurate forecast of underwater target echo and dynamically analog echo etc. The result has advantages of super-precision and calculating at a high-speed. It adapts to forecasting target echo engineering.

\section{References}

[1] FAN Jun, TANG Weilin. The planar element method for computing target strength (TS) of sonar[J]. Technical Acoustics,1999(sup):31-32. 
[2] FAN Jun. Study on Echo Characteristics of Underwater Complex Targets[D].Shanghai: Shanghai Jiao Tong University. 2002.

[3] FAN Jun, TANG Weilin. Planar Elements Method for Forecasting the Echo Characteristics from Sonar Targets[J]. Journal of Ship Mechanics, 2012, 16(1-2) 171 180.

[4] FAN Jun, ZHU Beili, TANG Weilin, Modified Geometrical Highlight Model of Echoes from Nonrigid Surface Sonar Target[J]. Acta Acustics,2001,26(6).545-550.

[5] Harry L. Van Trees. Optimum array processing. Part IV of Detection, Estimation, and Modulation Theory. Wiley. 2002. Chapter 4.

[6] Vittorio Murino, Andrea Trucco. Three dimensional image generation and processing in underwater acoustic vision. IEEE. 2000.88(12): 1903-1946.

[7] SHI Keren, GUO Yumin, Phased array ultrasound imaging detection[M]. Higher Education Press. 2010.Chapter 3.

[8] JIANG Xingzhou, CHEN Xi, JIANG Tao, Torpedo Guidance Design Principles[M].Naval Engineering University Press. 2006.Chapter 5. 